


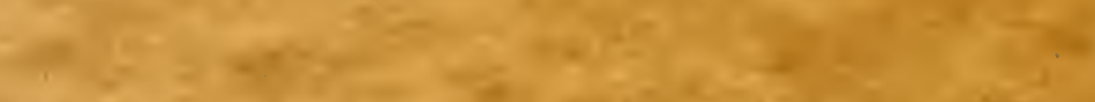

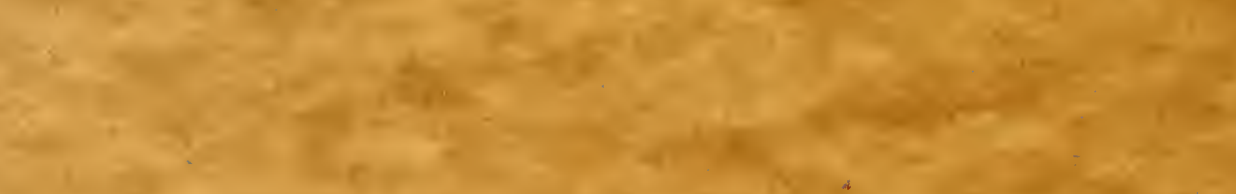

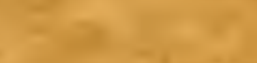

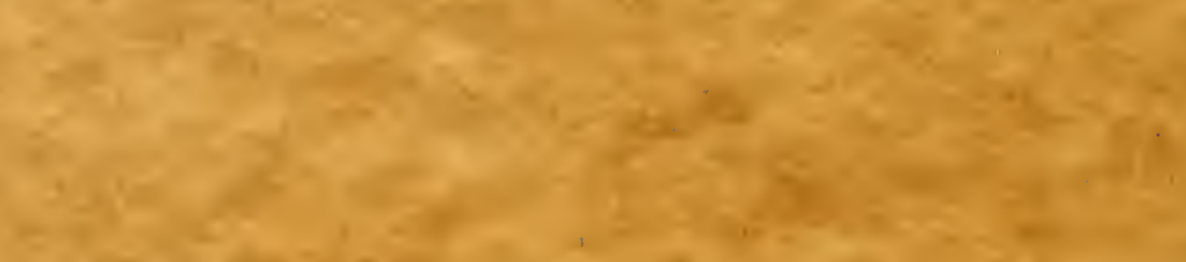

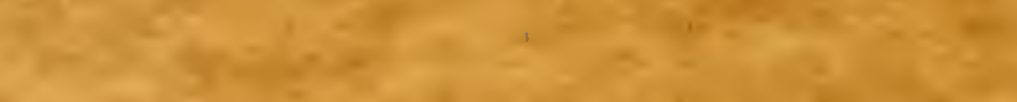

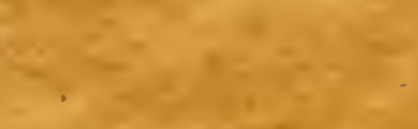

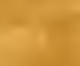

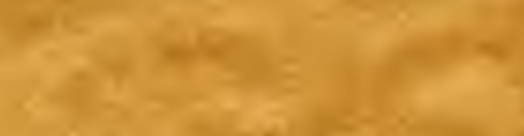

(a)

$+7$

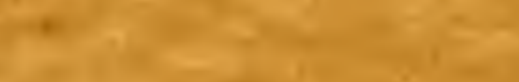

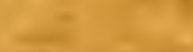

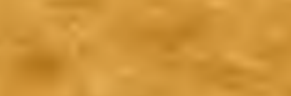

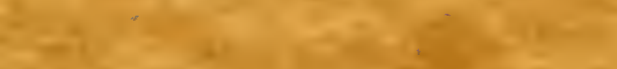

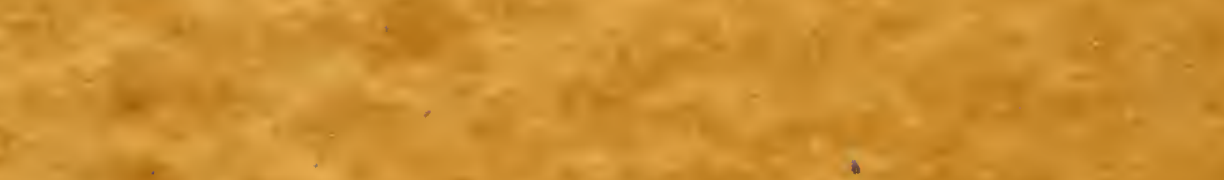

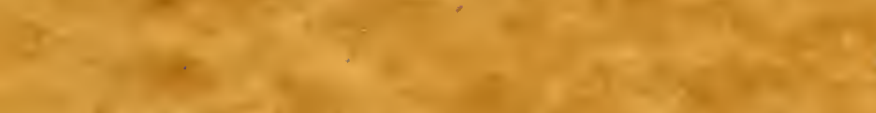

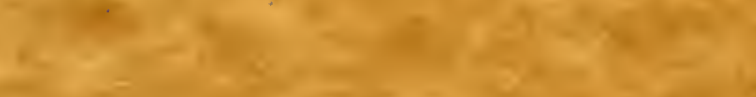

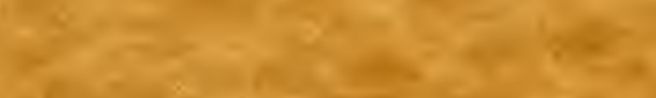

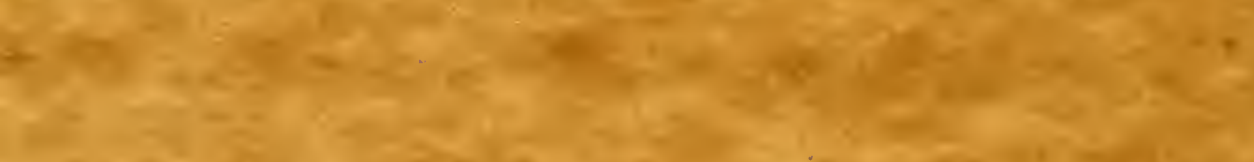

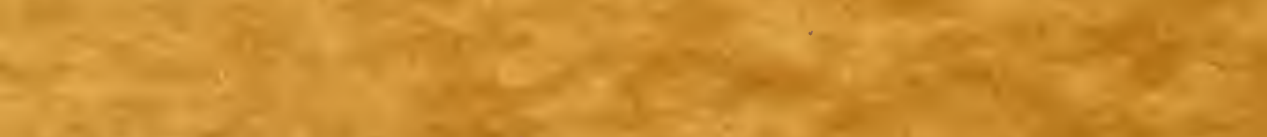
(7)

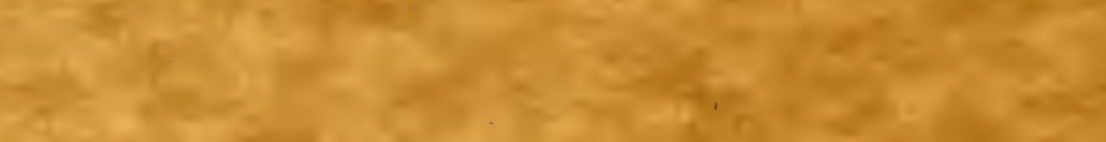

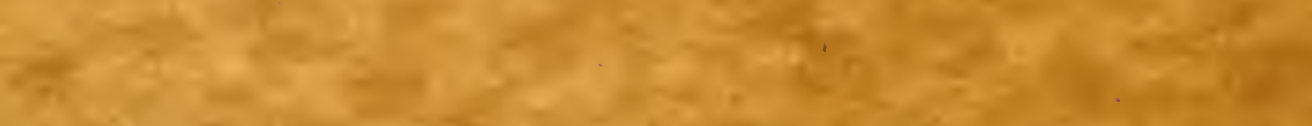

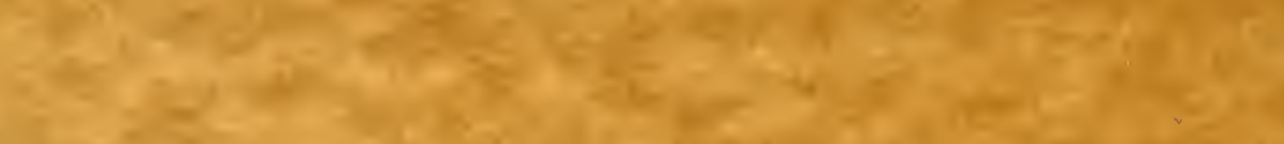

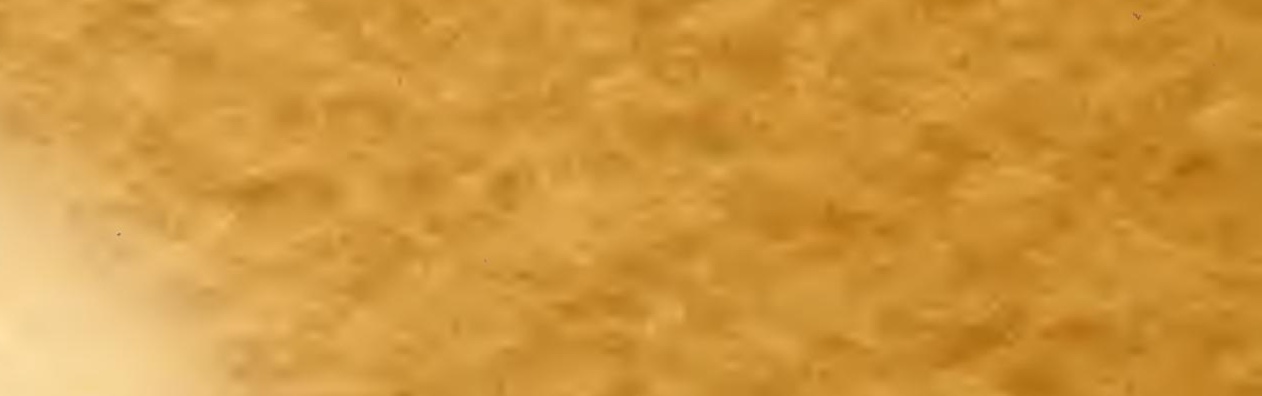


L ES

\section{Surcharges de Myrilène}

PAl:

\section{M. le Comte de Fayolle}

Extrait de la "Res'ue Philatélique Française", (numéro 257, spécial pour l'Exposition Philatélique Internationale de Paris, Juin 1913)

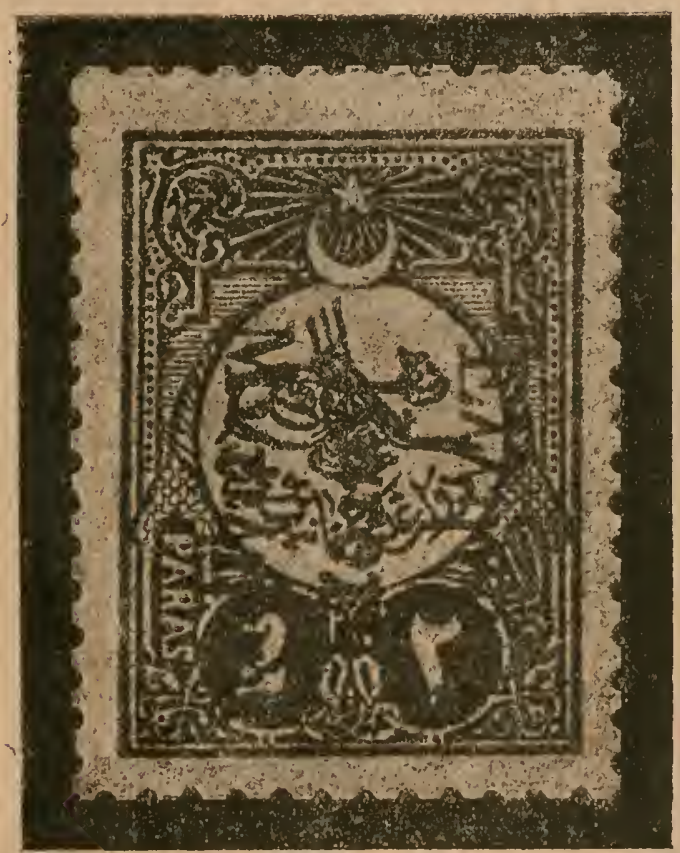

AMIENS

IMPRIMERIE YVERT ET TELLIER

1913

\section{Gift of GEORGE T'TURNER}





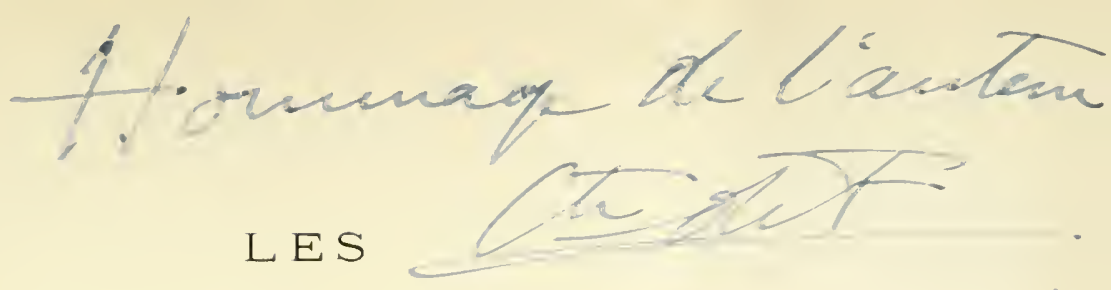

Surcharges de Mylinleme

P.II

\section{M. le Comte de Fayolle}

Extrait de la " lievue Philatélique Franẹaise", numero 257, spécial pour

l'Exposition Philatélique Internationale de Jaris, Juin 191:'

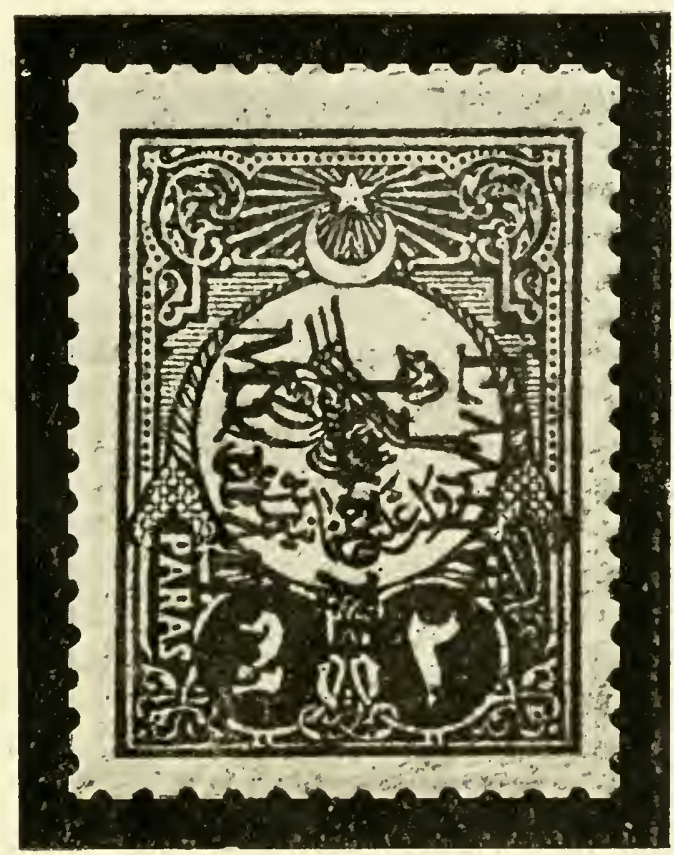

AMIENS

IMPRIMERIE TYERT ET TELI.IER 



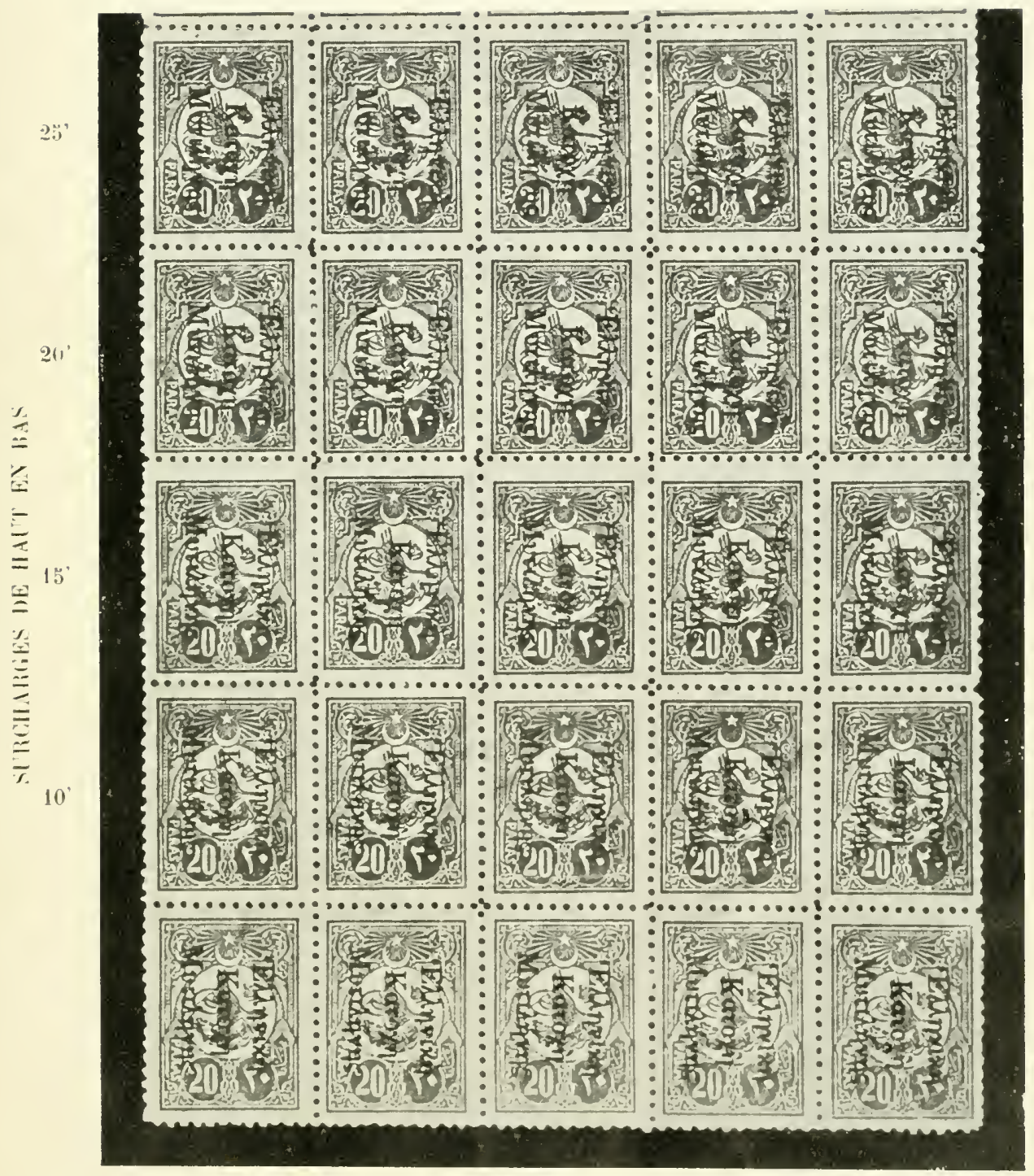





\section{E S}

\section{Surcharges de Myrinleme}

La quantité fabuleuse de fausses surcharges Eivr\%\%r,

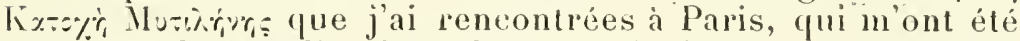
envoyées de Mélélin, d'Angleterre, d'Allemagne, d'Albènes et de Constantinople, le nombre tres minime des bonnes surcharges, et le prix souvent très élevé demandé non senlement pour les authentiques mais encore pour les faux, mont amené à faire une étude approfondie sur ces timbres qui m’intéressent au double titre de spécialiste de Gréce et de Turquie.

Cette surcharge fut exécutée à Mytilène par l'imprimerie "SALPINX", on forma avec des caractères soignés, destinés "l'impression de carles de visite, une composition typographique unique. Etait-ce la dimension réduite de sa presse, ou le manque de caractères qui obligea à exécuter uné planche ne pouvant surcharger que 25 timbres a la fois, je l'ignore, mais je pencherais pour la première hypothese, car les bords blancs des fenilles durent ètre enlevés, aucune leuille n’étant sorlie avec ces bords.

Chaque fenille de timbres tures destinée à la surcharge élait de 50 limbres, la composition typographique ne pouvait, à cause de ses dimensions, surchargor à cliaque passe que 25 timbres, soit une demi-fenille. Il existait néanmoins des deux rôtés entre les repéres ou les bords de la presse, "II jeu suflisant qui explique l'obliquité (remarquée parfois) de l'ensemble des surcharges par rapport a la verticale; de mème pour le jeu de haut en bas : j’ai remarqué que certains ensembles de 25 surcharges occupaient des positions diverses pour chaque demi-fenille.

La première moitié de la fenille surchargée, on relournait la fenille, et la seconde moitié se trouvait ainsi surchargée à l'envers. Il y a donc eu autant de surcharges "haul en 
bas" (celles de la $1 / 2$ inférieure) que de surcharges "bas en haut ". Limprimeur a dì avoir soin de présenter toujours les feuilles pour la première passe de facon que les timbres fussent dans leur position normale, car dans les diverses pièces, fenilles, blocs. que j'ai vus ou possède, la partie supérieure de la feuille est toujours surchargée: Bas en haut.

Grâce à l’obligeance de M. Nicolaïdès, auquel je suis redevable de nombre de renseignements des plus précieux pour cette étude et à celle de nombreux correspondants très sérieux, j’ai pu savoir de sources indiscutables (le Directeur des Postes, le Gonverneur de l'lle, divers ofliciers, etc:). le nombre à peu prés exact des diverses valeurs surchargées. Malheureusement le protocole ofliciel ne distingue pas pour certaines valeurs les divers "types ", ce qui serait très précienx, car, dans le nombre déja très restreint de certaines valeurs il y a des "types" dont la quantité surchargée a été extrêmement minime, par exemple les 133 (Y. et 'T.) tirés à 4 ? exemplaires!

Par un renseignement digne de foi, je savais depuis longtemps que les sureharges avaient été apposées sur une quantité de timbres dont la valeur faciale ne dépassait pas 155 Livres turques, la L. T. vaut environ $23 \mathrm{fr}$. 80 . Voici nue liste qui est d'accord avec ce chiflire.

\begin{tabular}{|c|c|c|c|c|c|}
\hline 18.500 & Piéces de & 2 & l'aras No & $Y+1$ & T. 1't \\
\hline 1.928 & ,. & 5 & m &, & $14 \%$ \\
\hline 1.731 & . & 10 & $n$ & , & 146 r.t $13: 3$ \\
\hline 18.961 & ," & 20 & , & , & 1.7 et 156 \\
\hline 4.330 & 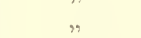 & 1 & Piastle & , & 148a et liza \\
\hline 220 & " & 2 & , & , & 133,1 \&9 et $1:$ ss \\
\hline 900 & , & $21 / 2$ & , & 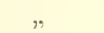 & 128 \\
\hline 133 & $n$ & 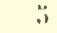 & , & , & $1: 31$ \\
\hline $9+$ & , & 10 & $"$ & " & $\left(127^{\circ}\right)$ el 152 \\
\hline 170 & laxes & 1 & . &, & +1 \\
\hline
\end{tabular}

Nayant jamais vu de 127 surchargé, je l'indique simplement à cause d'ouï-dires peu certains.

C'est le lendemain de l'occupation de l'Ile, soit le 9 Novembre 1912, que les surcharges furent ordonnées et exécutées, l'opération ne dura pas plus d'une heure.

Plus tard, le 23 Novembre (ou 6 Décembre style grec) on surchargea en nouvelles valeurs les quantités suivantes des timbres surchargés déjà le 9 Novembre :

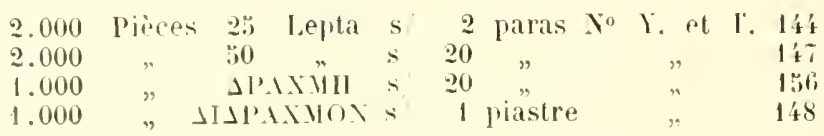


Il convient de faire observer que l'Ile de Mytilène possédait dans l'intérieur et sur la côte d’autres bureaux que ceux de Metelin, or les stocks de timbres tures de ces bureaus sont rentrés au bout de quelques jours dans la capitale de l'lle. Que sont derenus res timbres? on n'en sait rien! Cette "fuite "explique les réimpressions exécutées à Mytilène mème, et dont rous aurons à reparler.

On n'a pas eu non plus de nouvelles d'un paquet contenant pour 60 Livres turques, expédié par la Poste Centrale de Constantinople, arrivé 3 jours après l'occupation hellénique et saisi par les autorités grecques.

Tant aux bureaux tures de Smyrne que d'Ayvalik (ce dernier situé à une heure et demie de Mytilènt) il a été pendant quelque temps vendu (pour quel usage, on s'en doute, une quantité fabuleuse de timbres tures.

A la demande de M. Stellaké, Gouverneur de l'Ile, le Ministre des Postes de Grèce a fait retirer les deux anciens cachets turcs (cachet $n^{0} 1$ en turc et francais, cachet $n^{0} 2$ seu. lement en français, l'inscription turque ayant été enlevée par les Grecs), et les a lait envoyer ì Athènes, car les employés continuaient à oblitérer quantité de fausses surcharges.

Une enquète va paraît-il être ouverte contre ces agissements, mais les enquêtes Orientales me laissent plutòt sceptique ! !

Remarquons en passant que dans les faux on ne trouve pas jusquici les muméros 127, 133 et 158 (Y.et T.) car ces valeurs qui existaient en très petit nombre dans le stock de Mytilène (et qui ont été toutes surchargées) n’existaient plus ni à Smyrne ni à Ayvalik (oì elles étaient épuisées).

Voici le catalogue que j’ai dressé de toutes les surcharges :

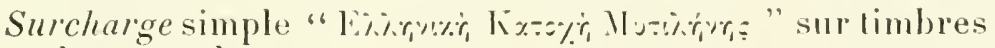
sans lettre arabe.

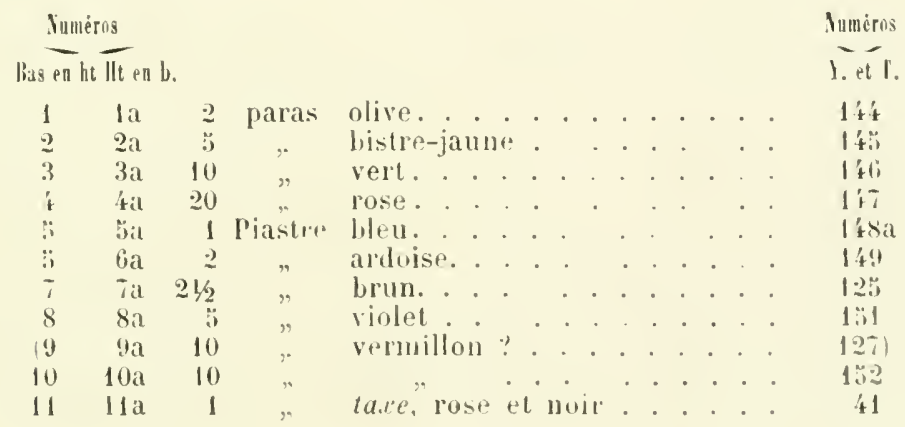




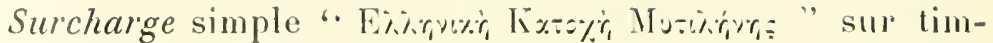
bres turcs avec lettre arabe:

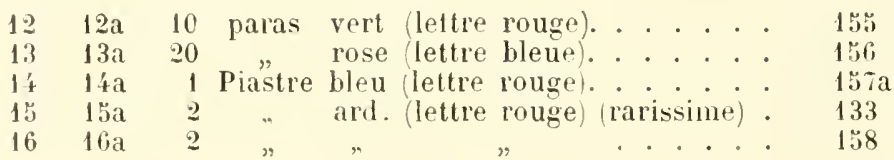

Mêmes timbres surchargés (sans lettre arabe) mais avec nouvelle valeur horizontale en lettres grecoues.

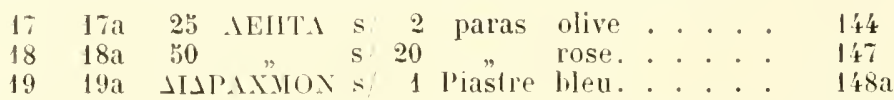

Idem, mais sur timbre avec lettre arabe (blenc) avec nouvelle valeur horizontale en lettres grecques.

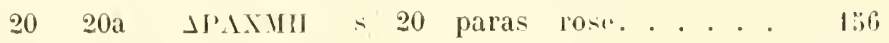

Je posside deux $n^{\circ}$ \& avee smcharge BLEUE, l'un surchargé $B . H$ ( $n^{\circ} 2$ dans la planche avec iota sans crochet). l'autre H. B. (nº 19 avec tan ayant la tète en forme de V). II a éte tiré 30 surcharges bleues environ à titre d'essai, les autorités trouvant cette teinte trop pâle firent exécuter la suite du tirage en noir ; jai aussi un $n^{\circ} 4$ portant une surcharge horizontale supplémentaire en blen-gris très pâle, cette surcharge très curieuse apposée à Plômarion, petit bureau de la còte ouest est la suivante :

$$
\begin{aligned}
& \text { E\\
INIKリ } \\
& \text { K. T'OXH } \\
& \text { IISIM }
\end{aligned}
$$

J'ai encore $u n n^{\circ} 12$, occupant le $n^{\circ} 22$ dans la planche avec double surcharge: l'une B. en H., l'autre H. en B., ces cinq pièces rarissimes sont rigoureusement authentiques.

Je possède un $n^{0} 17$ avec surcharge 25 \EIITA renversée, et un 20a avec double surcharge JP \X.MIl, les 2 empiétant l'une surl'autre presque à se confondre. Les surcharges de ces deux timbres sont authentiques, je le crois tout an moins.

Je n'ai pas connaissance d’utres variétés de ce grenre.

Nous allons passer aux pelites variétés dues à certaines erreurs: de composition ou dans les caracteres typographiques ou même dans la surcharge générale: E. K. MI.

En premier lieu je dois rappeler le mode l'impression de la surcharge : l'abord les 25 timbres du haut de la fenille (Bas en haut) ensuite les 25 restants (Haut en bas).

Nous retrouverons donc sur chaque feuille deux fois les mèmes erreurs, variétés, etc. forcément identiques ot situées 
symétriquement, seulement les unes seront B. II. les autres II. $B$.

En voici le tableau exact.

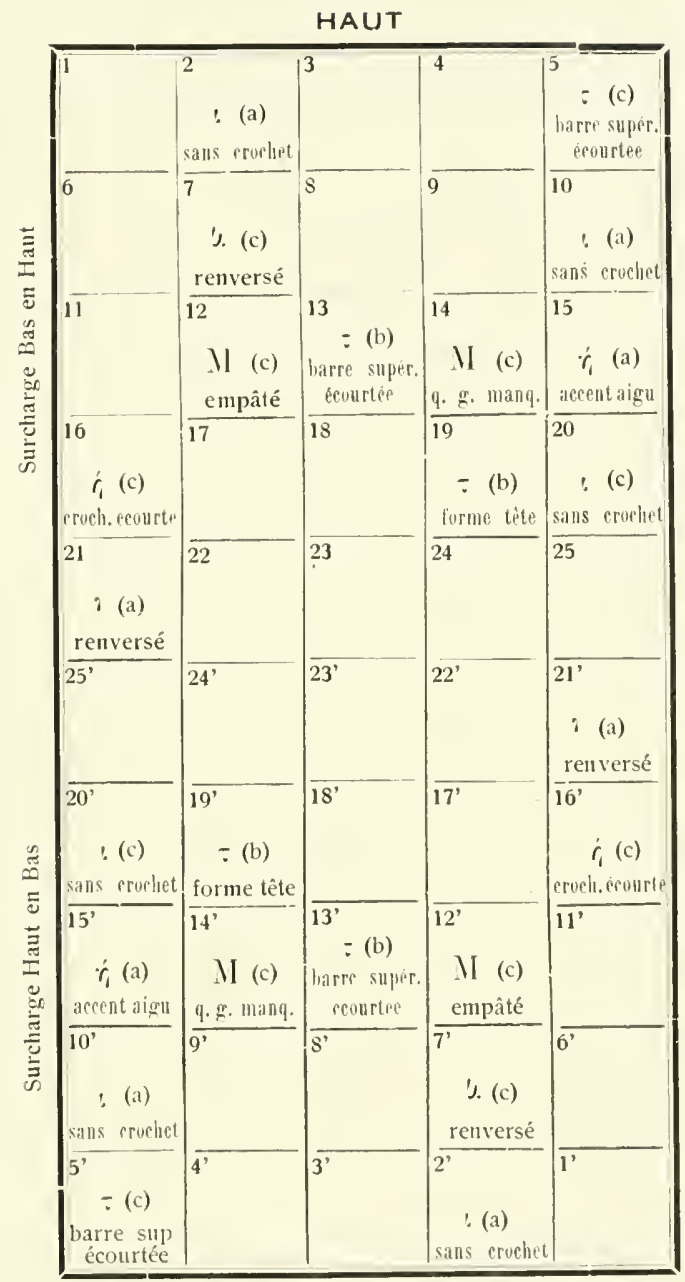

Pour plus de clarté nous indiquerons dans ce tableau par "a,, que l'erreur se trouve dans Eivr,\%\%, par "b,

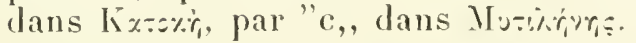

A remarquer que vu le nombre tris restreint des feulles surchargées, les caractères n'ont pas eu le temps de s'encrasser, ou des'abimer. Donc rejeter impitoyablement tous lestimbres qui ne sont pas rigoureusement conformes an type original. Je suis descendu jusquau plus infime détail 
de chaque lettre sur chaque timbre, tout autre caractere différant de ceux énumérés décèle une surcharğe fausse. Sur ce point je me permets d'ètre formel. J'ai eu entre les mains un nombre relativement considérable de timbres bons et un nombre colossal de faux et ne puis admettre de dérogation à ce principe

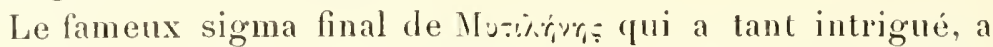
été choisi exprès par l’imprimeur dans une "série, différente de celle qui a fourni toutes les autres lettres; la forme de ce sigma est en effet très inusitée et a gèné considérablement nombre de faussaires.

'E La queue de l'accent ne dépasse jamais la barre supérieure de l'E qui est lui-mème très régulier partout.

$\therefore \quad$ Le $2^{i m} \%$ est plus bas que le premier infinitésimalement) dans tous les timbres sauí dans les $n^{\text {os }} 6.12,1$ ', 16 , 20 oin ils sont de mime hanleur. - Le 2 ime du 17 possède par exception une queue supérieure qui ne se termine pas en crochet.

$r_{1}$ Très régulier partout.

" Très régulier partout.

: ironversé dans le $n^{0} 21$ - - sans crochet infërieur dans : 2,10 .

\% Très régrulier partout

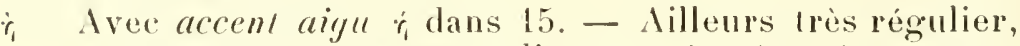
aver acceıt nettement distant et à mème écartement de la lettre.

Ii Très régulier.

* Assez régulier saul dans 1, 3, 5, 10, 11, 1'́, 23 où la queue supérieure est de forme carrée dans le haut.

- Ouene gauche de la barre horizonlale manque dans 13, et dans 19 la léte est en forme de $Y$.

= Très régulier.

\% Très régulier.

$\dot{r}_{1}$ Très régulier saul' les accents assez variés de forme, et à distance variable.

II No12: emprité, 'queues écourtées, barre de gauche plus grasse. Idem pour le 3 qui lui a les queues complètes. - Le no 1'́ a la quene supérieure de gauche absente.

- Assez régulier; le rentre des 13 et 22 est plus maigre; dans 7 la branche droite se termine par une ligne au lieu d'une larme.

- No 16: quene supérienre gauche un pen amincic. $N^{10} 5$ cette quencest absente et la lettre est déformée. - Nos 9 et 19 : la lettre est très maigre. 
: Très régulier - Sauf le 20: sans crochel.

ir" Très régulier. - Sauf le 16 : dont le crochet de $\dot{r}_{1}$ est écourté.

r. Très régulier saufle 7: renversé.

- La barre supérieure a généralement la lorme bien connue remontée; dans 12 et 15 elle est droite; dans 23 elle est droite et se termine un peu en goutte horizontale très droite et nette, ce qui la distingue des faux grossiers qui se terminent en larme tombunte.

D'une façon générale et absolue les lettres minuscules sont remarquablement alignées et ne se dépassent jamais en hauteur.

L’impression est loujours bonne, un peu bourrée, jamais gréle, l'encre légèrement brillante (les impressions grèles dénotent habituellement la réimpression).

Je ne doute pas qu'avec de l'habitude (surtout si on a dans l'œil les différents types de faux courants) on n'arrive à distinguer assez vite, presqu'au premier coup, la fausse surcharge de la bonne.

Cependant j'ai eu entre les mains quelques surcharges fausses venant d'une maison tristement célèbre d'Athènes: M. Nicolaïlès m'en a confié une autre série; enfin j’en ai moi-même acheté; or, certaines étaient tellement bien faites que seules les mesures au $10^{\text {mo }}$ et au $20^{\text {mø }}$ de millimètre que je vais indiquer ci-dessous ont pu me léceler leur nonauthenticité.

D'ailleur's d'une façon générale, bien peu de faux résistent aı micromètre! Personnellement je n'en connais pas.

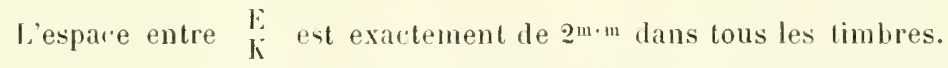

$$
\begin{aligned}
& \text { liespace entre } \mathbb{k} \text { est exactement de } 19 \text { dixıèmes dans tous les } \\
& \text { il timbres. }
\end{aligned}
$$

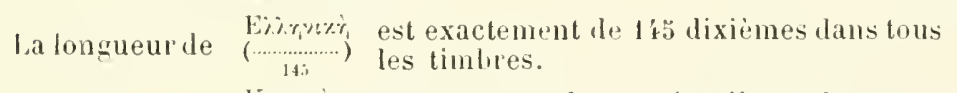

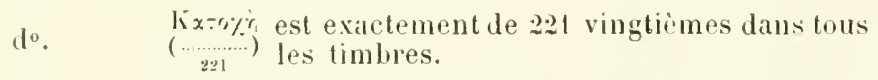

$$
\begin{aligned}
& \text { 10. Marivror est exactement de } 159 \text { dixièmes dans }
\end{aligned}
$$

sauf dans 1'́ et 18: 156 dixièmes, 13:313 vingtièmes à cause de l'écourtement des queues gauche haut et bas de M ; et 12: 157 dixièmes.

La hauteur totale de la surcharge entre le haut de E et le bas de $M$ est exactemenl pour lous les timbres de 235 vingtièmes de millimètre.

Je sais de bonne source que l'imprineur des surcharges 
a fait, avec les caractères qui lui avaient servi la première fois, une réimpression dont je parlerai plus loin. Or n'étaient ces critériums "dimensions" il serait presque impossible de découvrir cette réimpression, sauf à comparer certaines lettres, mais je garde pour moi ces dernières cartonches, comme ultime critérium.

Pour finir je vais donner quelques indications (qui m'ont été utiles dans plusieurs cas) sur les très diverses positions yulofrent entre eux les trois mots grecs constituant la surcharge. Crâce à ces positions on arrive rapidement à voir quelle place dans la planche doit occuper n’importe quel limbre isolé; ceci acquis, il devient relativement facile en comparant avec un étalon ou mème avec les photographies de voir si la sulcharge proposée est bonne, car dans chaque timbre il y a un petit quelque chose qui lui est particulier et que jamais aucun faussaire n'arrivera à imiter parfailement.

$1^{\circ}$ Te 16 à 25 on peut faire passer une ligne it peu près verticale par: la lre barre de $k$ et la 2 " de $\| l$.

A

K Pour les autres timbres l'obliquité de la ligne Al est absolument ii valiable.

B

2o) EnIre E et

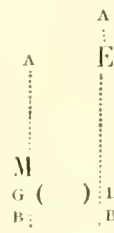

il y a des variés infinies d'ecart li ll.

$3^{\circ}$, Entre E el K $\mathrm{E}$

liciat est insigniliant dians les nos 14, I7 K et 18 .

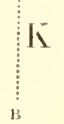

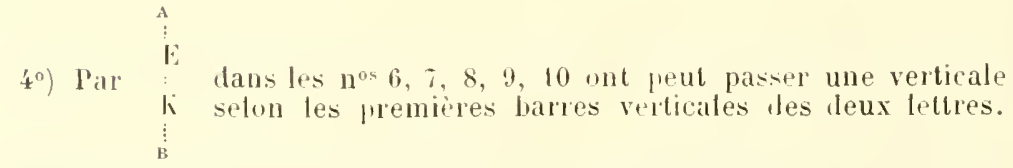

50) Pour tous les autres numéros la verticale passant frar la barre verticale de $k$ traverse environ en son milieu la lettre $E$ :

$$
\begin{aligned}
& \text { A } \\
& \vdots \\
& \text { li } \\
& \vdots \\
& \text { B }
\end{aligned}
$$

II. de Lormais qui habite Nytilène, et m'écrit frófuemment, me permet grâce à son extrème obligeance et 
gràce à la sirreté parfaite de ses somrces el de ses propres constatations "visuelles", de donner les précisions suivantes:

C'est le commandant Mélas, gouverneur militaire de l'Ile, qui a donné l'ordre de surcharger le stock de timbres tures saisis à la poste le 9/22 Novembre 1912. Le commandant Mélas avait alor's pleins pouvoirs sur l'Ile, militairement et administrativement.

Le chiffre de la valeur faciale totale des timbres surchargés que me donne M. de Lormais, diffère sensiblement de celui que j’indique plus haut, ce chiffre correspond d'ailleurs (à peu de chose près) avec celui que j’avais reç en Décembre. M. le Lormais me le donne pour 325 Livres turques, celui indiqué antérieurement, par M. Travlo, était de 322 L. T., or à ce moment $M$. de Lormais donnait le chiffre approximatif maximum de $200 \mathrm{~L}$. T., depuis j'avais recul de diverses sources, dont certaines indiscutables. tantôt 155 tantôt 325 livres, je m'en étais tenu pour diverses raisons que je erovais bonnes au chiffre de $155 \mathrm{~L}$. T. Je vais expliquer comment je crois ne pas m ètre trompé et comment les denx chiffres de M. de Lormais ne sont qu'en apparence discoldants.

J'ai dit plus haut que l'on ignorait ce qu'étaient devenues les $60 \mathrm{~L}$. T'. expédiées par la direction centrale des Postes de Constantinople à son bureau de Métélin avant l'occupation grecque, mais arrivées deux jours après cette occupation et saisies par les autorités grecques; que l'on ignorait aussi le destin du stock revenu des autres bureaux de l'lle, or un correspondant me disait il $r$ a trois mois (incidemment) (ju’on évaluait approximativement la valeur de ce stock à $100 \mathrm{~L}$. T.

Or: $155+60+100=315$, nous ne sommes done pas loin de 325 !

Il n'est pas moins certain qu’il n'y a eu le 9/22 Novembre 1912 que 155 L. T. de surchargées, et que la composition des caractères a été brouillée immédiatement après. Quid des 160 ou 170 restantes ? Voila non explication que je crois bonne, car elle est corroborée par maints renseignements épars, venant de toutes sortes de sources concordantes, et surtout par mon étude minutieuse de la lechnique des surcharges mèmes:

L’imprimeur de la surcharge première a, sur la demande de la poste elle-mème, refait mne composition de caracléres aussi semblable que possible ì la première il n'a naturellement pas pu arriver a me identilé absolue, jen ai expliqué déjà la raison) el il a exćculé une sorte de réimpression. Celle 
réimpression a-t-elle été officielle ou non, je l'ignore, ce qui est certain, et M. de Lormais me le confirme, c'est qu'aussitôt après il fut dressé un procès-verbal signé par le directemr de l'imprimerie, le directeur des postes el diverses autorités, interdisant au-dit imprimeur d'exécuter d'autres surcharges sur des timbres turos.

Enfin j’ai trouvé à maintes reprises des surcharges qui m'ont donné beaucoup de mal: mèmes lettres, dimensions à pell près exactes, mème encre, mème aspect général etc., et cependant légères diflérences et surtout impossibilité de sitıer chaque pièce dans la planche complète; chose bizarre, les surcharges de cette sorte ne portaient que sur les timbres turos autres que les 2. 5 et 10 piastres (lesquels n'existaient yue dans le stock de Méłélin et non dans l'envoi de Constantinople ni dans le stock des antres villes de l'lle), on ne trouve dans ces trois valeurs que: ou des pièces rarissimes incontestablement authenticues on des faux très ralactérisés et de provenances bien définies.

Bien que ces réimpressions offrent quelque chose d'un peu ofliciel, je ne les admettrai comme " Reimpressions Olliciclles "que lorsque :

$1^{\circ}$ J'aurai en mains au moins une fenille complète d'une de ces réimpressions, afin d'avoir une base certaine d'expertise.

$2^{\circ}$ Lorsque j'anrai la certitude que ces réimpressions on été effectrées avec l'assentiment formel des autorités et non sur l'iniliative plus ou moins louche d'un employé de la poste, et cela d'autant mieux : que M. de Lormais me confrrme après plusieurs autres qu'une instruction est ouverte contre certain employé pour agissements au moins bizarres, et qu'enfin, comme je l'ai dit, interdiction a été faite à l’imprimeur de faire de nouvelles surcharges.

Junque là je considérerai ces jièces comme des faux.

M. de Lormais n'accorde aucune valeur aux "surchareres en lettres grecques : " en effet, me dit-il, la poste ellenème, l'imprimenr non plus », ne savent sur combien de limbres ces nouvelles surcharges ont été appliquées ; "en somme c'est l'imprimenr qui - sur la demande de la l'oste bien entendu - a fait celte opération à sa guise ".

M. de Lormais n'ayant à l'appui de son allégation aucune pièce olficielle je réserve ce point jusqu’à plus ample informé, tout en penchant pour son opinion.

Il me dit aussi que la quantité des surcharges se décompose ainsi: "à peine 50 pièces du 2 piastres (avec lettre (1rabe) 90 seulement du 10 piastres, environ 150 dı 1 pi. Taxe, le rester réparti pour quantités à peu près d’égale valeur pour cliaque timbie n. 
Ce qui indique la rareté fabuleuse des authentiques, c'est ce qu'ajoute mon aimable et très documenté correspondant:

"Il n'existe que 21 séries complètes par suite du manque des timbres de 2 et 10 piastres; en eflet, les autorités ont acheté 34 pièces de cette dernière valeur, un négociant en a acheté 35 pièces dont il demande fort cher, mais ne possédant aucune autre valeur il n'a pas de séries. Restent donc 21 timbres qui se trouvent dans 21 séries complètes ".

Suit la nomenclature de ces séries, avee le nombre détenu par chacun des heureux possesseur's et les lieux ou elles se trouvent. J'ai pu me rendre dernièrement acquéreur de deux d'entre elles

En ce qui concerne les cachets oblitérants, M. de Lormais confirme ce que j'ai dit plus haut.

In fine il ajoute: " $\Lambda$ Métélin mème une personne venant de S.... a importé quantité de faux et je puis allirmer que si nous arons sur la place à peine pour $60 \mathrm{~L}$. T. de "bons" nous avons pour plus de $500 \mathrm{~L}$. T. de faux!!"

Cela confirme, quoique à mots couverts, ce que je vais dire sur le tratie de certains individus.

II n'y a pas trois jours dans un carnet d'échange de la S. F. de T. se trouvaient plusieurs timbres de Mytilène, ils étaient tous faux, un enfant les aurait expertisés; renseignements pris : c'était encore un certain Y. qui les avait mis en circulation! ! ainsi d'ailleurs que tous ceux expédiés à diverses reprises à l'Union Timbrologique d'Echanges.

MI. Yvert et Tellier viennent de fermer lenrs colonnes d'annonces à toute une clique qui abuse vraiment, ce n'est qu'en démasquant les voleur's qu'on arrivera à se débarrasser d'enx. Je venx bien croire que quelques rares pèchent par ignorance, tant pis pour eux: on ne se mèle pas d'ètre marehand de timbres lorsqu'on n'y connaît rien et que l'on enrosse sa clientèle chaque fois qué l'occasion s'en présente.

Vula rareté extrème de cette émission de Mrtilène, beancoup ont dii se laisser tenter par l'appàt de prix très élevés.

En tous cas, depuis deux mois, il m'a paru bizare et je ne suis pas le seul) de constater que certains faux d'une facture bien cléfinie, et toujours la mème selon chaque provenance, vinssent toujours des mimes sources.

J'ai réuni sullisamment de renseignements, de lettres, de preuves pour me faire une opinion très nette.

Ln tous as, je tiens de source indisculable que l'un de cenx qui a inondé Paris, l'Ingletere el Berlin d'un stock de faux, est venu ì Mélélin muni de timbres tures, a werché a faire surcharger son lot et a cité mis à la porte de l'imp rimerie 
Lesbos; que cette mème personne à qui à phısieurs reprises on a renvoyé ses faux) a toujours déclaré par écrit qu’ils élaient bons tout en se refusant à les signer pour des raisons ridicules (attendu qu'il signe habituellement ses envois), que par un bien singulier hasard il a renvoré ces jours-ci une série bonne, signée "moins un timbre "lequel était hasard encore plus curieux)... faux !! Cette personne aurait (d'après des renseignements recus ces jours-ci) congédić son secré. taire lequel aurait une grosse part de culpabilité !!!

Détail bizarre: une personne amie de ce monsieur,a passé dernièrement à Paris et à Bruxelles, ce que cette personne ofl'rait était faux...

D’autres personnes, avant. elles, une rraie raison sociale philatélique, ont inondé le marché de fanx: l'une de Constantinoples'est particulièrement distinguée. Une autre d'Athènes déjà trop connue, arec l’intermédiaire de certains : A..u, L..o, a envové des faux à tous les marchands, j’en ai mème reçu un envoi bizarle: agréable mélange de peu de "rrais " el de beancoup de faux; deux autres personnages: l'un Arménien: A..n, l'autre Girec: E....s, ont fait le mème trafic. On me signale, avec faux à l'appui, un autre Athénien: M. M. B...s, el à Smyrue : B. C...ou.

Un certain directeur de poste étrangère à Métélin possède me quantité stupéfiante de surchargés, l'ex-directeur de la poste grecque (actuellement destitué) a prèté la main à des tripatoullages très curieux.

Enfin je viens d’ètre avisé res jours-ci (15 Juillet que ce mèmo Directem de Poste étrangère de Métélin vient-de faire deux envois a Paris de faux : l'un de 3.000 , l'autre de 7.800 fr's! Nous cherchons à qui ces envois ont été faits.

Je me permets de trouver bien piteuse la facon dont collectionneurs et marchands honnètes, victimes périodiquement de faussaires éhontés: parisiens, grees, levantins, etc.. etc.. hésitent à prérenir bien haut lous les philatélistes chaque fois qu'ils trouvent un pot... aux timbres.

J'admets que certains faussaires le soient par ignorance ?. ils n'en sont pas moins coupables.

J'ai, à tort ou à raison, été mèlé à des discussions entre divers marchands; plusieurs ont dì reconnaitre qu'ils avaient rendu comme authentiques, par erreur, certains Mytilène faux, en tous cas je puis dire très carrément que seule la signature Nicolaïdès n'a jannais été apposée, à ma connaissance du noins, que sur drs timbres rigonreusement authentiques. et que Nicolä̈dis arant. nombre de fois. cul recours a mes faibles lmmières, sön opinion et la mienne ont toujours cété 


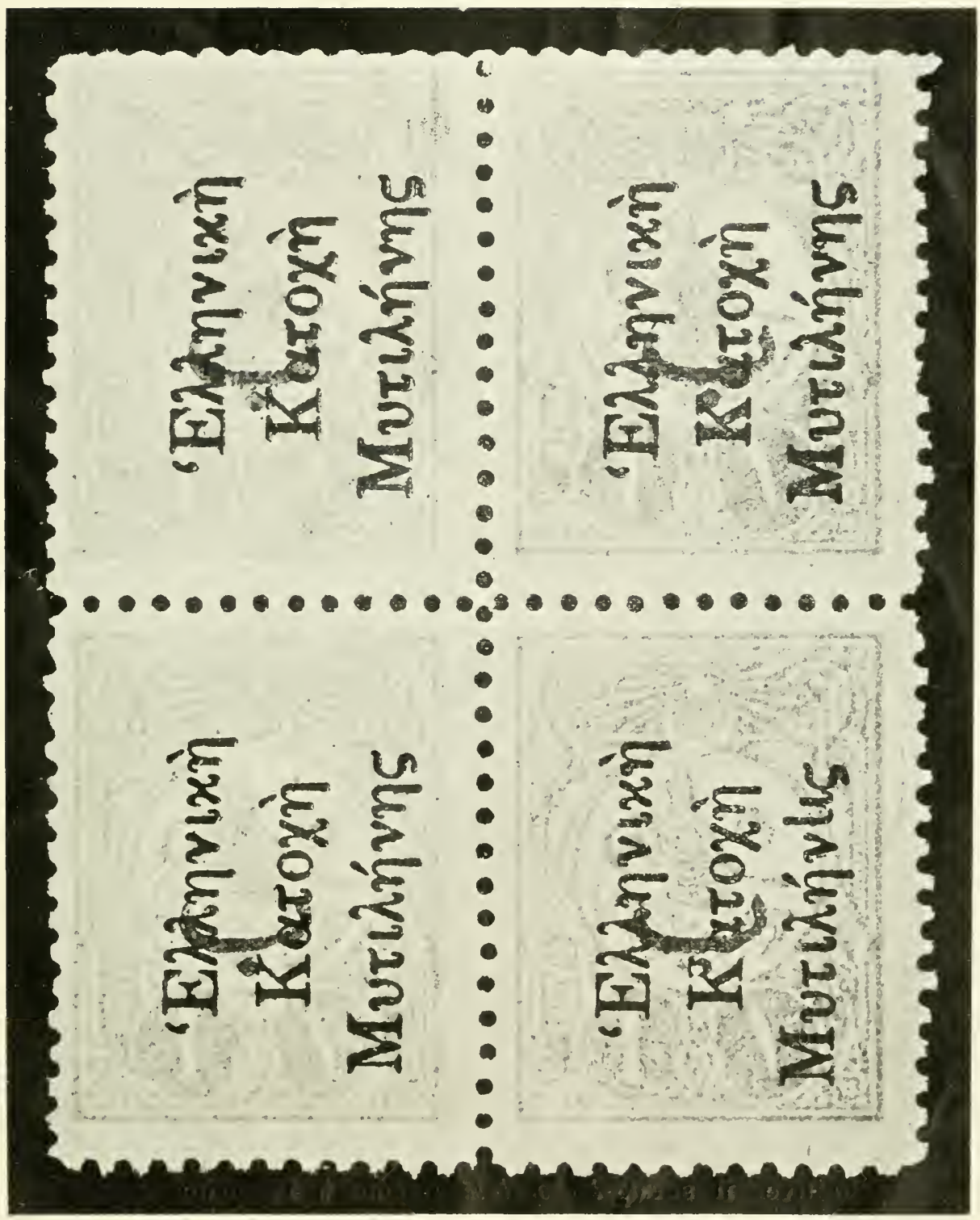


d'accord. Je dois à son obligeance la communication d'un

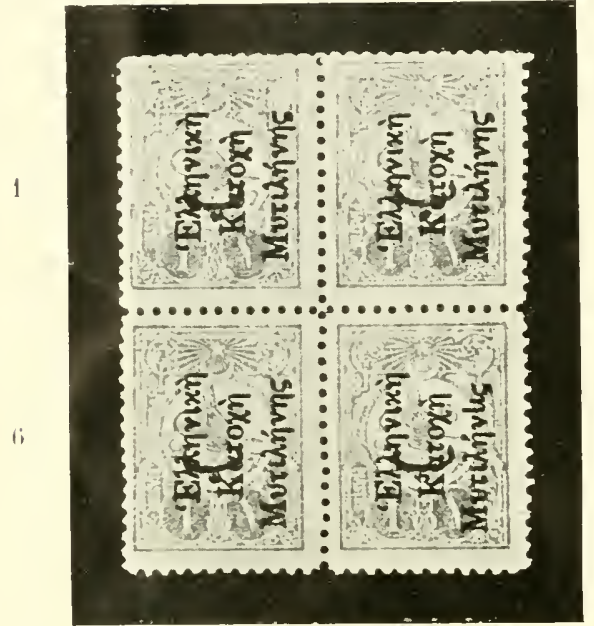

bloc des une piastre avec lettre arabe Ce bloc dont la couleur très hlene a permis une bonne renue 2 photographique de la surcharge est composé des timbres: 1, 2, 6 et 7 de la fenille. C'est donc le bloc formant l'angle supérienr ganche. On remarquera quil contient l'erreul du èta renversé timbre 7) et que l'iota dn timbre $\mathbf{n}^{\circ} 2$ n'a pas de rrochet.

Si quelguim de mes lectems ponvait me fournir une fenille ou tout an moins une demi-fenille d'un 1 piastre quelcongue, je serais acheteur au plus haut prix.

Le petite tache blanche que l'on peut observer sur la partic supérieure dı "k " est un défaut photographique et n'existe pas sur le timbre (limbre $n^{0} 6$.

En dehors de ce blor je regrette de ne pomroir dommer i mes lerteurs (indulgents je l'espère) des photographies plus lisibles que celles de mes denx feuilles complètes, ce n'est fu’à force d’écrans et de retirages que je suis arrivé all très médiocre résultat ci-joint, mais le 2 paras es! olive aver beancoup de jame dans la coulemr), le 20 paras est rose!

A propos des deus feuilles représentées, el qui ne doivent pas ètre communes entières, je puis en dire la provenance : elles ont été envoyées à M. Nicolaïlès par mo oflicier supérieur de la flotte grecque qui est précisément un de renx qui ont surveillé le tirage et ont défait le cadre et les coins de la composition typographicfue et brouillé tous les caractères. aussitôt l’impression terminée.

Or quiconque est au courant du métier d'imprimenr comprendra comment mème avec les mímes: cadre, coins, caractères, en reproduisant les mèmes espaces, en copiant le mienx possible toutes les dispositions de la composition primitive, il serait impossible (nême à l'imprimeur) de reproduire exactement les mèmes surcharges. J'ai pris l'avis de plusieurs imprimeurs, tous sont formels à cet égard. Ne serait.ce que parce que les caractères étant par leur composition mème (plomb, antimoine etr.) déformables, la pression 
des coins et du cadre ne peut matériellement être identique ; en outre quelle difficulté pour le faussaire le plus soigneux de remettre à sa place exacte chaque lettre! C'est grâce au changement imperceptible d'un iota et d'un tau que j'ai pu découvrir la non authenticité d'un 20 paras que m'avait obligeamment communiqué M. Paul de Smeth Ce timbre constituait le faux le plus dangereux de tous ceux que j'ai eu à expertiser, je me hâte de dire d'ailleur's que la presque totalité des fausses surcharges se décèle bien plus facilement.

Dernières nourelles (5 Aoùt). - Le Directeur de poste étrangère dont j'ai déjà parlé à deux reprises, s'est associé a un certain M. R...i, lequel a plusieurs procès à Alexandrie pour vente de faux.

Après le premier directeur, M. Saccas, révoqué à cause des faux Mytilène (et qui continue à Athènes sur les 10 pi. surtout), le second directeur de la poste vient d'ètre destitué, à cause parait-il des grees surchargés et non des faux.

Comte de Farolle.

4, Rue St-Florentin, Paris.

N.-B. - En raison de notre mantue de loisirs el du tres grand

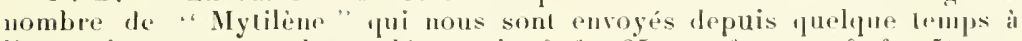

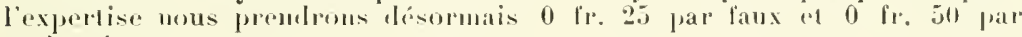
anthentique. 






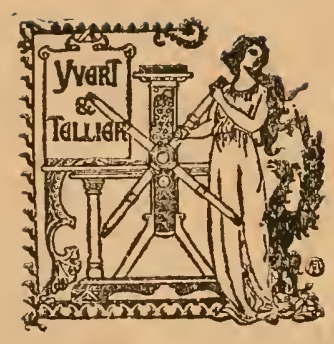


Pamphlet

Binder

Gaylord Bros., Inc

Makers

Syracuse, IN. Y.

PQT. JGN 21. 1908

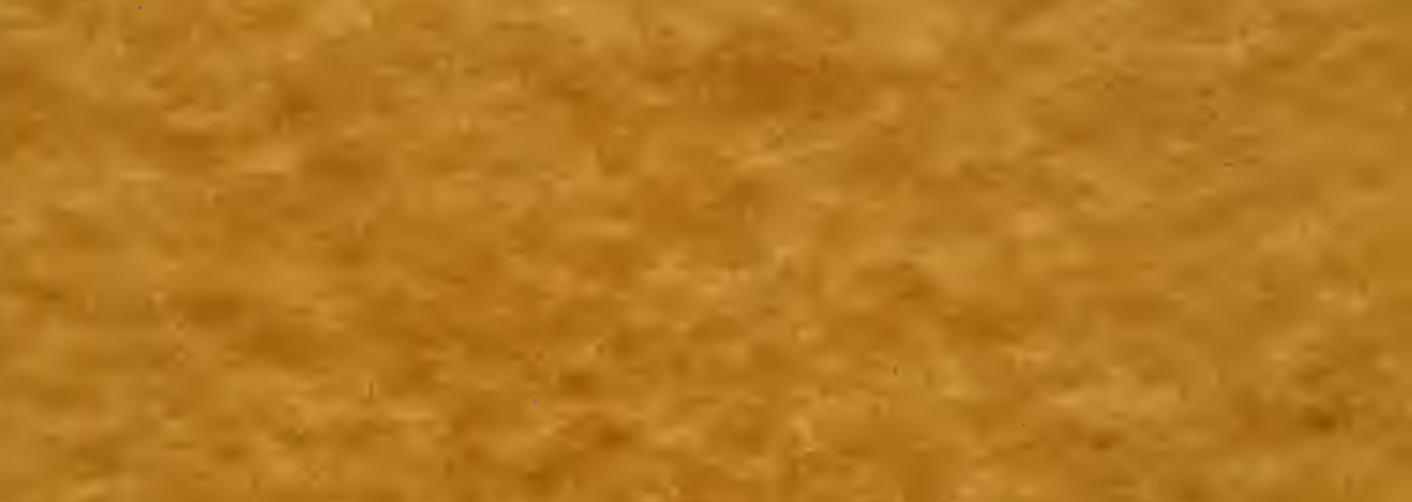
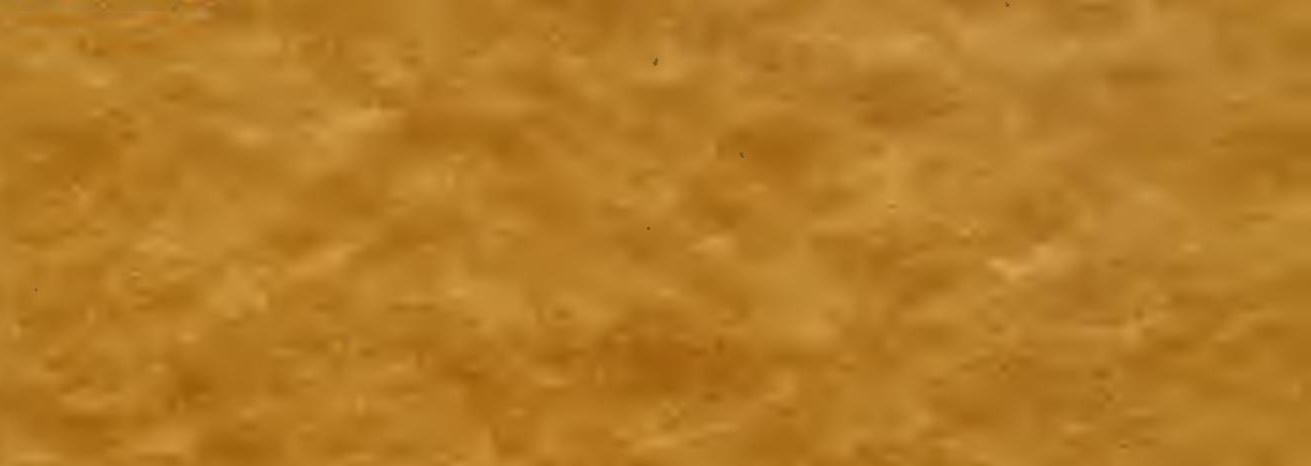

s.
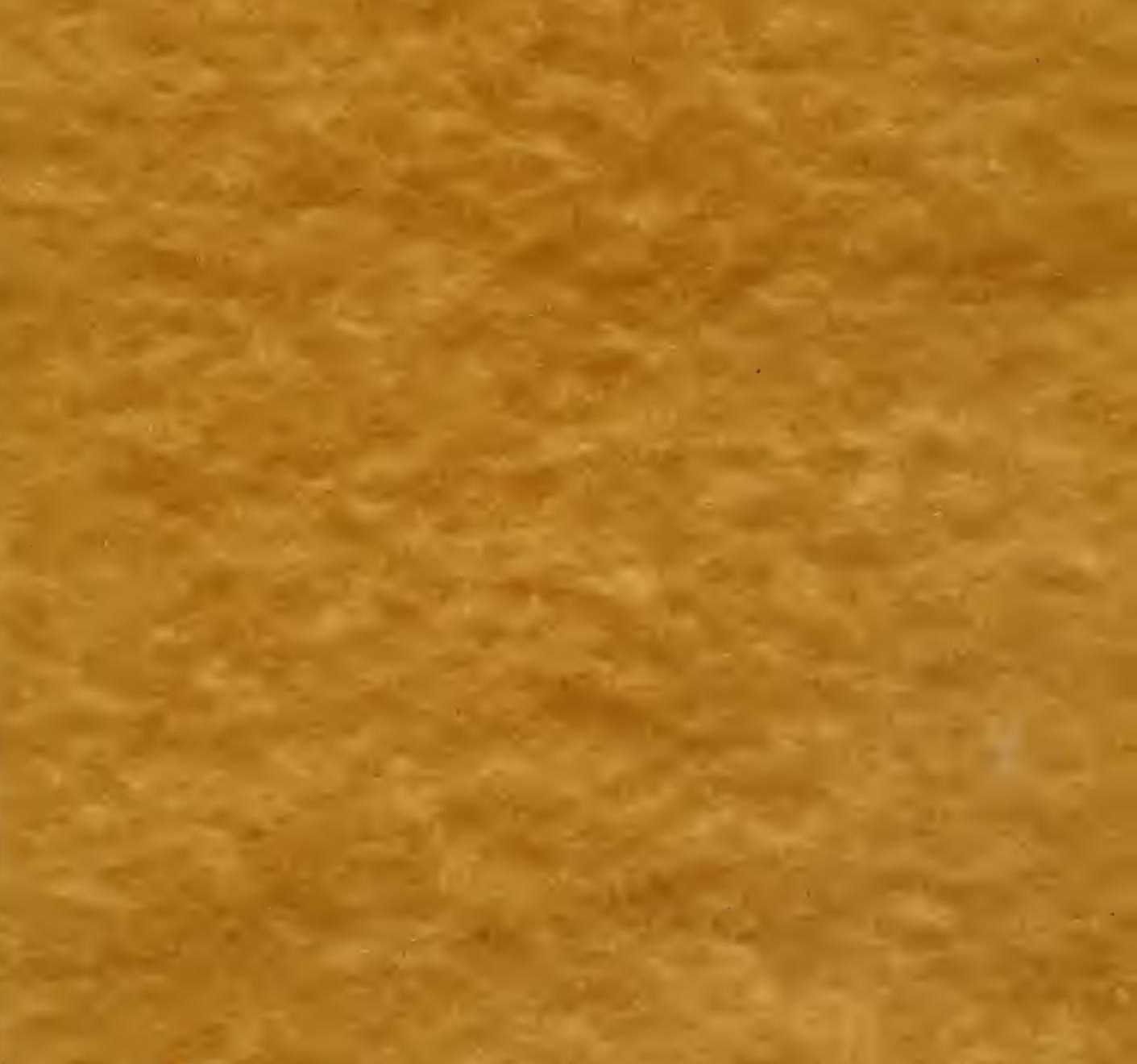
